\title{
Multi-GEM Detectors in High Particle Fluxes
}

\author{
P. Thuiner ${ }^{1,2, a}$, F. Resnati ${ }^{1, b}$, S. Franchino ${ }^{1}$, D. Gonzalez Diaz ${ }^{1,3}$, R. Hall-Wilton ${ }^{4}$, H. Müller ${ }^{1}$, E. Oliveri ${ }^{1}$, D. Pfeiffer ${ }^{1,4}$, \\ L. Ropelewski ${ }^{1}$, M. Van Stenis ${ }^{1}$, C. Streli ${ }^{2}$, and R. Veenhof ${ }^{3}$ \\ ${ }^{1}$ CERN, European Organization for Nuclear Research, 1211 Geneva, Switzerland \\ ${ }^{2}$ Technische Universität Wien, Atominstitut, Stadionallee 2, 1020 Vienna, Austria \\ ${ }^{3}$ Uludağ University, Department of Physics, 16059 Bursa, Turkey \\ ${ }^{4}$ ESS, European Spallation Source, 22100 Lund, Sweden
}

\begin{abstract}
Gaseous Electron Multipliers (GEM) are well known for stable operation at high particle fluxes. We present a study of the intrinsic limits of GEM detectors when exposed to very high particle fluxes of the order of $\mathrm{MHz} / \mathrm{mm}^{2}$. We give an interpretation to the variations of the effective gain, which, as a function of the particle flux, first increases and then decreases. We also discuss the reduction of the ion back-flow with increasing flux. We present measurements and simulations of a triple GEM detector, describing its behaviour in terms of accumulation of positive ions that results in changes of the transfer fields and the amplification fields. The behaviour is expected to be common to all multi-stage amplification devices where the efficiency of transferring the electrons from one stage to the next one is not $100 \%$.
\end{abstract}

\section{Introduction}

Gaseous Electron Multipliers (GEM) [1] are known to maintain stable gains when exposed to $6 \mathrm{keV}$ X-ray fluxes of at least $10^{6} \mathrm{~Hz} / \mathrm{mm}^{2}$ for a single stage device operated at a gain of about $10^{2}$ [2], and at least $10^{5} \mathrm{~Hz} / \mathrm{mm}^{2}$ for double stage devices operated at a gain of $2 \times 10^{4}$ [3]. For triple stage devices it was observed that at high fluxes of soft X-rays the effective gain as a function of the flux first increases and then decreases [4]. This effect was assumed to be due to space-charge effects resulting in field distortions in the detector. Considerable efforts have been made to reduce the back-flow of positive ions into the conversion volume of multi GEM detectors to reduce space-charge induced field distortions [5][6]. Further studies [7] show a decrease of the ion back-flow of a triple GEM detector due to ion space-charge when exposed to high X-ray fluxes. In sections 2 and 3 we will explain the experimental setup and methods used. Section 4 contains measurements and models describing the observed effects. Conclusions and outlooks are found in section 5 .

\section{Experimental setup}

A standard triple GEM detector with double-conical holes of $70 \mu \mathrm{m}(50 \mu \mathrm{m})$ outer (inner) hole diameter and pitch of $140 \mu \mathrm{m}$ in an active area of $10 \times 10 \mathrm{~cm}^{2}$ is used. The drift gap is $3 \mathrm{~mm}$ long, all other gaps are $2 \mathrm{~mm}$ in length. A schematic representation of the detector is shown in figure 1. All electrodes of the detector are powered either

\footnotetext{
a e-mail: patrik.thuiner@cern.ch

be-mail: filippo.resnati@cern.ch
}

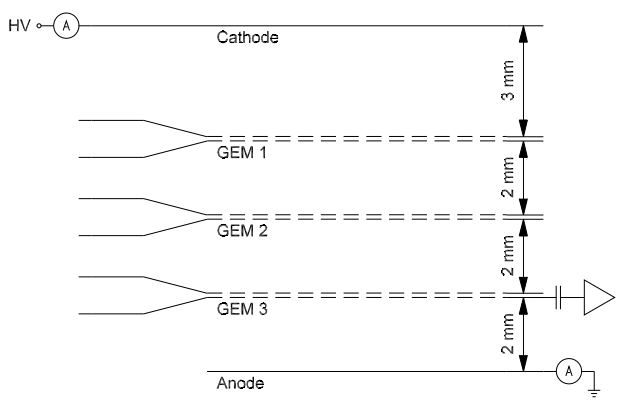

Figure 1: Schematic representation of the setup.

by resistor divider (results in figure 2) or individually (results in figure 3) using high current resolution power supplies with a resolution of $50 \mathrm{pA}$ for all stages. The drift cathode is powered individually for all results discussed in the following sections. The read-out anode is read with a pico-amperemeter (Keithley 6487) with a resolution of approximately $5 \times 10^{-5}$ times the monitored current. To facilitate event by event measurements of the signals, a decoupling capacitor of $2.2 \mathrm{nF}$ connects the bottom electrode of the third GEM to a charge pre-amplifier (ORTEC 142) and a shaper (ORTEC 572) chain connected to a Multichannel Analyzer (AMPTEK MCA-8000D). The detector is continuously flushed with an $\mathrm{Ar} / \mathrm{CO}_{2} 70 / 30$ gas mixture at $5 \mathrm{~L} / \mathrm{h}$. Collimated $8 \mathrm{keV} \mathrm{X-rays} \mathrm{of} \mathrm{about} 1 \mathrm{~mm}^{2}$ beam diameter generated by a copper X-ray tube are used to produce the primary ionisation charges of approximately 290 primary electrons in the conversion volume. The X-ray 
flux is adjustable by changing the X-ray tube intensity or using calibrated attenuators.

\section{Experimental methods}

The observed effects are checked to be unrelated to global effective gain variations, e.g. due to changing of ambient parameters. A reference position $P_{r e f}$ is continuously irradiated with an Fe-55 source. The peak position of the $5.9 \mathrm{keV}$ peak in the pulse height spectra is continuously monitored when the shutter of the X-ray tube is closed. All electrodes are powered for at least one hour before starting the measurements to ensure stability of the currents monitored. Charging-up effects [8] are excluded as the origin of the observed behaviour by waiting until the currents $I_{i, o n}$ and the peak position of the $8 \mathrm{keV}$ peak in the pulse height spectra are stable after starting the irradiation at low flux, and by repeated measurements going from low to high X-ray flux and vice versa. The currents collected at the drift cathode, GEM electrodes and read-out anode are calculated as $I_{i}=I_{i, o n}-I_{\text {beamoff }}$ by measuring the currents $I_{\text {beamoff }}$, taking into account the charge collected when the detector is continuously exposed to low flux X-ray of the Fe-55 source as well as leakage currents from electrodes to ground, and $I_{i, o n}$ during irradiation with high X-ray fluxes of the $\mathrm{Cu} \mathrm{X}$-ray tube.

The effective gain is calculated as $G_{e f f}=I_{A} /\left(R n_{p} e\right)$ with the current $I_{A}$ collected at the read-out anode, the estimated interaction rate $R$, the number of primary electrons $n_{p} \approx 290$ for the gas mixture used and the electron charge $e$. The ions per primary electron are calculated in a similar way as $G_{i o n}=I_{C} /\left(R n_{p} e\right)$ with the current $I_{C}$ collected at the drift cathode. The ion back-flow is defined as the ratio $I B F=I_{C} / I_{A}$ of the currents collected on cathode and anode.

\section{Measurements}

As shown in figure 2 the effective gain, as a function of the X-ray flux is stable up to a few $\mathrm{kHz} / \mathrm{mm}^{2}$ for the nominal effective gain studied. Increasing the flux results in a slight rise of effective gain, which becomes prominent when increasing the flux above a few hundred $\mathrm{kHz} / \mathrm{mm}^{2}$. When reaching a flux of about $1 \mathrm{MHz} / \mathrm{mm}^{2}$ the effective gain reaches a peak before decreasing significantly at a flux of the order of $10 \mathrm{MHz} / \mathrm{mm}^{2}$.

The number of ions per primary electron show an inverse behaviour up to $1 \mathrm{MHz} / \mathrm{mm}^{2}$, with the value decreasing. When the effective gain reaches the peak and start decreasing again, the ions further decrease, with the slope of the curve getting flatter with increasing X-ray flux.

The ion back-flow starts decreasing significantly when the effective gain increases. At a flux of the order of $4 \times 10^{6} \mathrm{~Hz} / \mathrm{mm}^{2}$, the ion back-flow reaches a plateau.

The observed change in effective gain is strongly dependent on the nominal effective gain of the detector (Fig. 3).

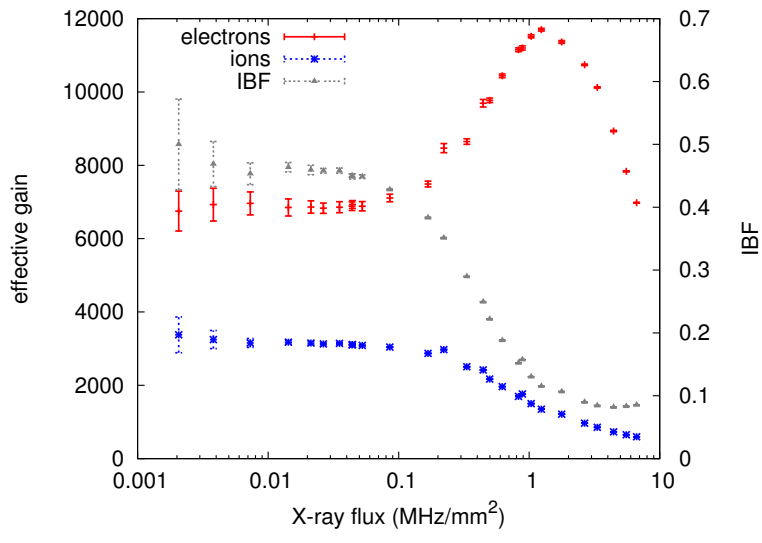

Figure 2: Dependence of the effective gain, the number of ions per primary electron, and ion back-flow on flux of $8 \mathrm{keV} \mathrm{X}$-rays for a nominal effective gain of approx. $7 \times 10^{3}$ in $\mathrm{Ar} / \mathrm{CO}_{2} 70 / 30$ gas mixture.

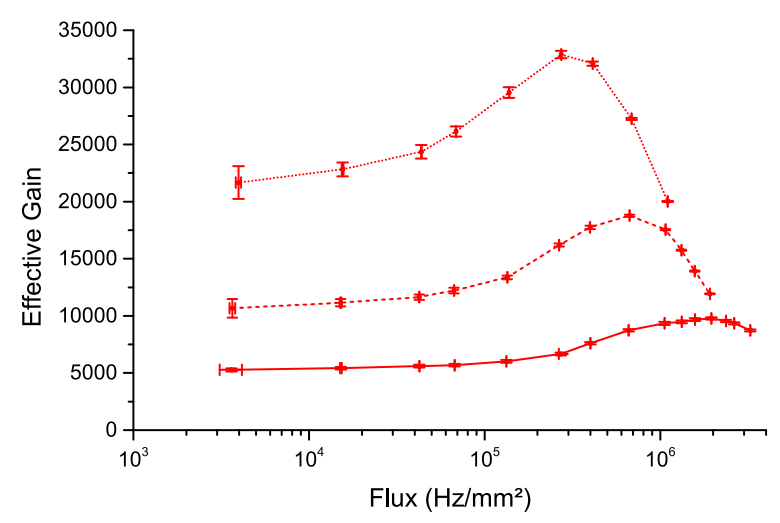

Figure 3: Dependence of effective gain on $8 \mathrm{keV} \mathrm{X-ray}$ flux for nominal effective gains of $5 \times 10^{3}, 10^{4}$ and $2 \times 10^{4}$.

Since the effect is related to the charge density in the detector, increasing the nominal effective gain will lead to the appearance of the observed effects at lower particle fluxes while a decreased gain will shift the effect towards higher fluxes.

The behaviour of the triple GEM detector is simulated using the Finite Element Analysis software package COMSOL Multiphysics ${ }^{\circledR}$ [9]. The electron and ion distributions in the detector are described macroscopically by numerically approximating the solutions of a set of partial differential equations of second order: the drift and diffusion equations for electrons and ions, and the Poisson equation for the potential distribution. Electron transport coefficients are computed using Magboltz 8.97 [10], and the amplification is calculated with the Townsend coefficient corrected for Penning transfer [11]. The ion mobilities are taken from [12]. A two-dimensional axialsymmetric geometry is used to simulate the effect in a triple GEM detector. The modelled GEMs therefore only contain a single GEM hole, with the holes of all three GEMs perfectly aligned. 


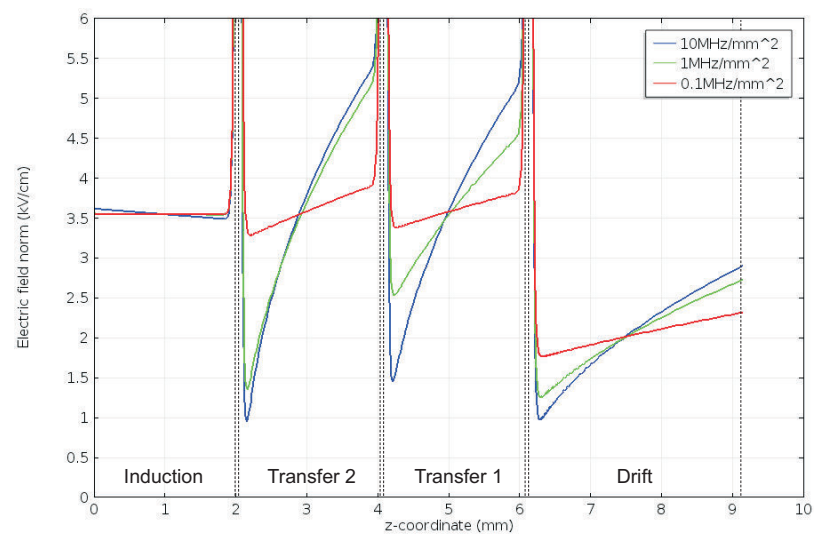

Figure 4: Computation of the field distortions in the transfer gaps for X-ray fluxes of $0.1 \mathrm{MHz} / \mathrm{mm}^{2}$ (red), $1 \mathrm{MHz} / \mathrm{mm}^{2}$ (green) and $10 \mathrm{MHz} / \mathrm{mm}^{2}$ (blue) along the hole axis. The nominal fields are $2 \mathrm{kV} / \mathrm{cm}$ in the Drift gap and $3.5 \mathrm{kV} / \mathrm{cm}$ in the other gaps.

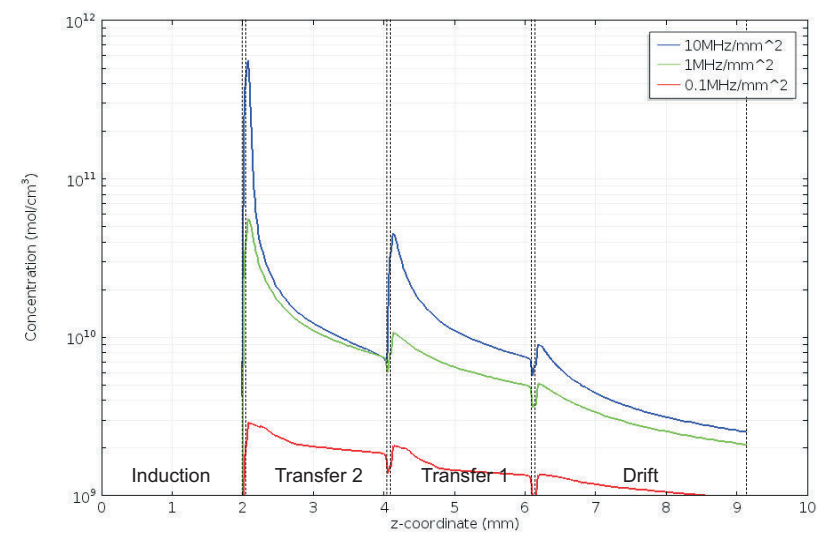

Figure 5: Computation of the ion concentrations in the detector for X-ray fluxes of $0.1 \mathrm{MHz} / \mathrm{mm}^{2}$ (red), $1 \mathrm{MHz} / \mathrm{mm}^{2}$ (green) and $10 \mathrm{MHz} / \mathrm{mm}^{2}$ (blue) along the hole axis.
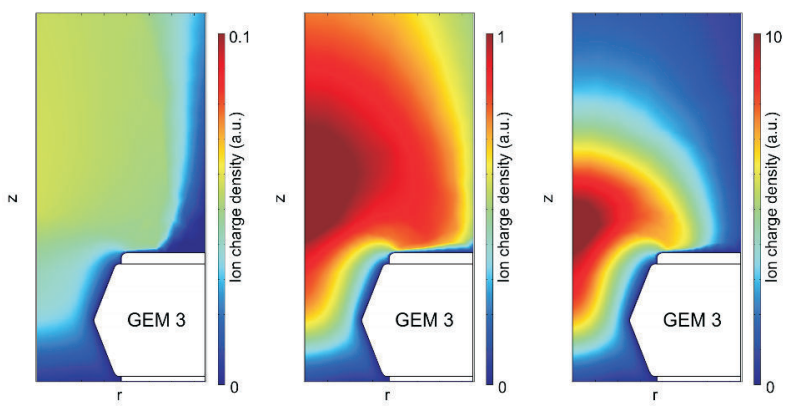

Figure 6: Computation of the ion charge density close to the bottommost GEM for X-ray fluxes of $0.1 \mathrm{MHz} / \mathrm{mm}^{2}$ (left), $1 \mathrm{MHz} / \mathrm{mm}^{2}$ (center) and $10 \mathrm{MHz} / \mathrm{mm}^{2}$ (right) along the hole axis.

The ions created in the GEM holes during the electron avalanches drift upward into the transfer and conversion volumes. The dynamic equilibrium of production and

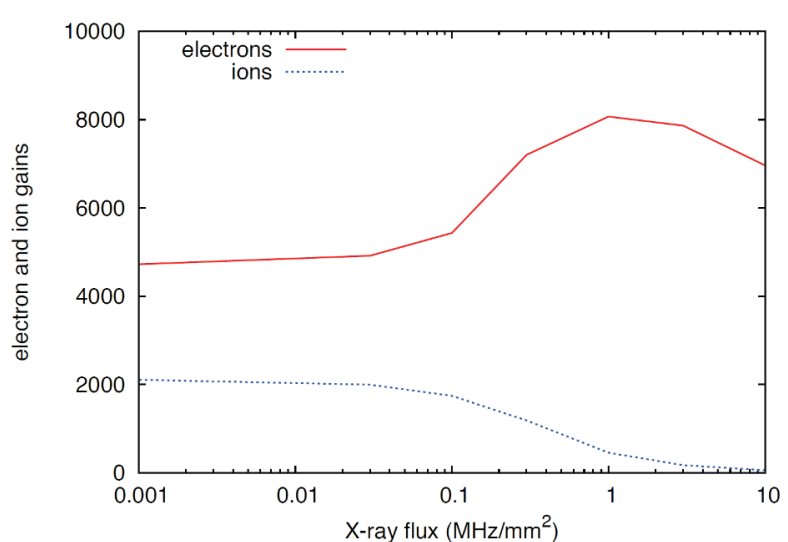

Figure 7: Computation of effective gain and number of ions per primary electron for different X-ray fluxes at a nominal effective gain of approx. $5 \times 10^{3}$.

evacuation results in an accumulation of positive ions in these volumes. This leads to a steady space-charge distribution that modifies the electric field. For increasing $\mathrm{X}$-ray flux the fields get distorted in a way that the fields close to the top of the GEM electrodes are reduced and the fields close to the bottom electrodes are increased (figure 4), with the nominal field as the average over the length of the gap. The effect becomes stronger for each successive stage of amplification, with the smallest distortion in the Drift gap and the largest in the Transfer 2 gap. The distortions in the Induction gap are minimal due to an absence of ions in this region.

The electron collection and extraction efficiencies of a GEM are strongly dependent on the fields applied above and below the GEM, respectively [13]. This results in two effects: first, both the collection and extraction efficiencies for electrons are increased. With the efficiencies for electrons not being $100 \%$ for the nominal fields applied, this results in an observable increase in effective gain up to the point where the collection and extraction efficiencies reach $100 \%$. Secondly, at the same time the collection and extraction efficiencies for ions are decreased. This leads to a significant reduction of the ion back-flow. Effective gain and ions per primary electron for the simulations are calculated as already described in section 3 . As can be seen in figure 7, the simulations are semi-quantitatively comparable to the measurements shown in figure 2 .

After reaching the effective gain peak at approximately $1 \mathrm{MHz} / \mathrm{mm}^{2}$, space-charge effects in the amplification regions become dominant. With the ion extraction decreasing significantly, the ions start accumulating close to the top of the GEM holes. Due to the presence of the ions the fields are further decreased, additionally reducing the ion extraction. Ions generated during an avalanche are now accumulating in great number in the GEM holes, thus reducing the fields and decreasing the effective gain of the detector. This effect is simulated and can be seen in figure 5. At the same time the transfer fields are modified in a way, such that the evacuated ions get collected by the top 
electrode of the GEM in increasing number, leading to a reduced ion back-flow. This effect can be seen in figure 6 . While the effects described are observed in all stages of the triple-GEM detector, the last GEM foil is the one dominating the behaviour of the detector.

\section{Conclusions}

Space-charge effects for high fluxes of $8 \mathrm{keV} \mathrm{X-rays} \mathrm{in} \mathrm{a}$ $\mathrm{Ar} / \mathrm{CO}_{2} 70 / 30$ gas mixture were studied. At high particle flux ion space-charge modifies the transfer fields and the amplification fields such that, as a function of the flux, the effective gain first increases and then decreases. The ion back-flow is decreasing with increasing particle flux. The field distortions are dependent on the flux and the nominal effective gain of the detector. Increasing the nominal effective gain leads to the appearance of the observed effects at lower X-ray flux while a decreased gain shifts the effect towards higher flux. The effects are modelled for standard triple-GEM detectors and quantitatively understood. Further systematic studies of the energy resolution will be soon published elsewhere.

\section{References}

[1] F. Sauli, Nucl. Instr. Meth. A 386, 531 (1997)

[2] J. Benlloch et al., IEEE Trans. Nucl. Sci. 45, 234 (1998)

[3] A. Bressan et al., Nucl. Instr. Meth. A 425, 262 (1999)

[4] P. Everaerts, Master Thesis, Gent University (2006)

[5] F. Sauli, L. Ropelewski, P. Everaerts, Nucl. Instr. Meth. A 560, 269 (2006)

[6] A. Breskin et al., Nucl. Instr. Meth. A 553, 46 (2005)

[7] M. Ball, K. Eckstein, T. Gunji, Journal of Instrumentation 9, C04025 (2014)

[8] M. Alfonsi et al., Nucl. Instr. Meth. A 671, 6 (2012)

[9] Comsol multiphysics ${ }^{\circledR}$, http://www . comsol.com

[10] S. Biagi, Nucl. Instr. Meth. A 421, 234 (1999)

[11] Özkan Şahin, T.Z. Kowalski, R. Veenhof, Nucl. Instr. Meth. A 768, 104 (2014)

[12] H.W. Ellis et al., Atom. Data and Nucl. Data Tables 17, 177 (1976)

[13] S. Bachmann et al., Nucl. Instr. Meth. A 438, 376 (1999) 